\title{
A QPSO based Damping Controller for STATCOM
}

\author{
Amin Safari \\ Department of Electrical Engineering, \\ Azarbaijan Shahid Madani University, Tabriz, Iran
}

\begin{abstract}
The objective of this paper is to investigate the enhancement of damping the low frequency oscillations via tuning of static synchronous compensator (STATCOM) damping controller. The problem of STATCOM based damping controller is formulated as an optimization problem according with the time domain-based objective function which is solved by a quantum-behaved particle swarm optimization (QPSO) technique that has fewer parameters and stronger search capability than the classical PSO. The performance of the designed controller is demonstrated through nonlinear time-domain simulation and some performance indices studies in comparison with designed CPSO, genetic algorithm (GA) and phase compensation based STATCOM controllers.
\end{abstract}

Keywords: STATCOM, Quantum Particle Swarm Optimization, Damping Controller, Genetic Algorithm

\section{Introduction}

Flexible AC transmission systems (FACTS) technologies have received a large amount of attention recently. The main objective of FACTS devices is to control power flows and improve voltage characteristic in a power system but, due to their fast response, FACTS can also be used for power system stability enhancement through improved damping of power oscillations [1]. Through the adjustment of bus voltage, phase difference between buses, and transmission line reactance, FACTS devices can cause a considerable increase in transfer power limits during steady-state operating.

One of the members of FACTS family is Static synchronous compensator (STATCOM) which is connected in shunt with the system. It injects the bulkiness reactive elements of conventional static VAR compensator by a synchronous voltage source. The performance of STATCOM is to generate a controllable AC voltage source behind a transformer-leakage reactance so that the voltage difference across the reactance produces active and reactive power exchange between the STATCOM and the transmission network. Several trials have been reported in the literature to dynamic modeling of STATCOM in order to design suitable controllers for power flow, voltage and damping controls [2]. In Ref. [3], linearized PhillipsHeffron model of a power system installed with a STATCOM is presented. The author has not presented a systematic method for designing the damping controllers. Moreover, no effort seems to have been made to design the STATCOM damping controller optimal parameters, in order to achieve at a flexible damping controller. Fuzzy logic based controllers have been used for controlling a STATCOM by Morris et al., [4]. The performance of such controllers can further be improved by adaptively updating their parameters. Although using the robust control methods $[5,6]$, the uncertainties are directly introduced to the synthesis, but due to the size of large power systems the order of resulting controller will be very large in general, which is not feasible because of the computational economical difficulties in practical implementing. 
The particle swarm optimizer is a population based meta-heuristic algorithm, which utilizes the swarm intelligence generated by the cooperation and competition between the particles in a swarm and has emerged as a useful tool for engineering optimization [7-9]. Unlike the other heuristic techniques [10], it has a flexible and well-balanced mechanism to enhance the global and local exploration capabilities. However, the main disadvantage is that the classical PSO algorithm is not guaranteed to be global convergent. In order to overcome this drawback and improve optimization synthesis, in this paper, a QPSO technique is applied for optimal tuning of a STATCOM based damping controller for damping of power systems low frequency oscillations. The QPSO algorithm is depicted only with the position vector without velocity vector, which is a simpler algorithm and the results show that QPSO performs better than standard PSO and is a promising algorithm due to its global convergence guaranteed characteristic [11-14]. A problem of interest in the power industry is the mitigation of power system oscillations. These oscillations are related to the dynamics of system power transfer and often exhibit poor damping. The main motivation of this work is to damp out the electromechanical oscillations using the SATCOM with proposed damping controller in a power system.

In this study, the problem of robust STATCOM based damping controller design is formulated as an optimization problem and QPSO technique is used to solve it. The aim of the optimization is to search for the optimum controller parameter settings that improve the dynamic system performance. The performance and effectiveness of the proposed controller is demonstrated through nonlinear time-domain simulation and some performance indices to damp electromechanical oscillations under different operating conditions. Results evaluation show that the proposed damping controller achieves good robust performance for a wide range of operating conditions and disturbance.

\section{PSO and QPSO}

\subsection{Particle Swarm Optimization}

Particle swarm optimization algorithm, which is tailored for optimizing difficult numerical functions and based on metaphor of human social interaction, is capable of mimicking the ability of human societies to process knowledge. It has roots in two main component methodologies [8]: artificial life and evolutionary computation. The PSO technique can be used to solve many of the same kinds of problems as GA, and does not suffer from some of GAs difficulties. This optimization method has been found to be robust in solving problem featuring non-linearing, non-differentiability and high-dimensionality.

The PSO begins with a swarm of random solutions particles in a D-dimension space. The ith particle is depicted by $\mathrm{X}_{\mathrm{i}}=\left(\mathrm{x}_{\mathrm{i} 1}, \mathrm{x}_{\mathrm{i} 2}, \ldots, \mathrm{x}_{\mathrm{iD}}\right)$. Each particle in swarm keeps track of its coordinates in hyperspace, which are associated with the fittest solution it has obtained so far by any particle in the population. The value of the fitness for particle $\mathrm{i}$ (pbest) is also stored as $\mathrm{P}_{\mathrm{i}}=\left(\mathrm{p}_{\mathrm{i} 1}, \mathrm{p}_{\mathrm{i} 2}, \ldots, \mathrm{p}_{\mathrm{iD}}\right)$. The global fittest solution of the PSO keeps track of the overall best value (gbest), and its location, obtained so far. PSO consists of, at each step, changing the velocity of each particle toward its pbest and gbest according to equation (1). The velocity of particle $\mathrm{i}$ is represented as $\mathrm{V}_{\mathrm{i}}=\left(\mathrm{v}_{\mathrm{i} 1}, \mathrm{v}_{\mathrm{i} 2} \ldots \mathrm{v}_{\mathrm{iD}}\right)$. The position of the ith particle is then updated according to equation (2) $[9,15]$.

$$
\begin{aligned}
& v_{i d}(t+1)=w \times v_{i d}(t)+c_{1} r_{1}\left(P_{i d}-x_{i d}(t)\right)+c_{2} r_{2}\left(P_{g d}-x_{i d}(t)\right) \\
& x_{i d}(t+1)=x_{i d}(t)+v_{i d}(t+1)
\end{aligned}
$$


Where, $P_{i d}$ and $P_{g d}$ are pbest and gbest. The $w$ is the inertia weight parameter. Constants $c_{1}, c_{2}$ are cognitive and social co-efficients, respectively, and $r_{l}, r_{2}$ are random numbers between 0 and 1. A larger inertia weight factor is used during initial exploration of its value is gradually reduced as the search proceeds.

\section{2. Quantum-Behaved Particle Swarm Optimization}

The main disadvantage is that the PSO algorithm is not guaranteed to be global convergent. In classical PSO technique, a particle is depicted by its position vector $x_{i}$ and velocity vector $v_{i}$, which determines the trajectory of the particle. The dynamic behavior of the particle is widely divergent form that of the particle in CPSO systems in that the exact values of $x_{i}$ and $v_{i}$ cannot be determined simultaneously. In quantum world, the term trajectory is meaningless, because $x_{i}$ and $v_{i}$ of a particle cannot be determined simultaneously according to uncertainty principle. Therefore, if individual particles in a PSO system have quantum behavior, the PSO algorithm is bound to work in a different fashion $[13,14]$. In the quantum model of a PSO called here QPSO, the state of a particle is depicted by wave function $\Psi(x, t)$ instead of position and velocity. Employing the Monte Carlo method, the particles move according to the following iterative equation:

$$
\begin{array}{ll}
x_{i}(t+1)=p+\beta \cdot \mid \text { Mbest }_{i}-x_{i}(t) \mid \cdot \ln (1 / u) & \text { if } k \geq 0.5 \\
x_{i}(t+1)=p-\beta \cdot \mid \text { Mbest }_{i}-x_{i}(t) \mid \cdot \ln (1 / u) & \text { if } k<0.5
\end{array}
$$

Where, $u$ and $k$ are values generated according to a uniform probability distribution in the range $[0,1]$; the parameter $\beta$ is called Contraction-Expansion coefficient which can be tuned to control the convergence speed of the particle [12]. In the QPSO, the parameter $\beta$ must be set as $\beta<1.782$ to guarantee convergence of the particle. Thus, the equation (3) is the fundamental iterative equation of the particle's position for the QPSO. Moreover, unlike the PSO, the QPSO needs no velocity vectors for particles at all, and also has fewer parameters to control (only one parameter $\beta$ except population size and maximum iteration number), making it easier to implement. The experiment results on some well-known benchmark functions show that the QPSO described by the following procedure has better performance than the PSO [11] where Mbest, called mean best position, is defined as the mean of the pbest positions of all particles. i.e.,

$$
\text { Mbest }=\frac{1}{N} \sum_{d=1}^{N} p_{i d}(t)
$$

Trajectory analyses in [13] demonstrated the fact that convergence of the PSO algorithm may be achieved if each particle converges to its local attractor, $p$ defined at the coordinates:

$$
p=\left(c_{1} p_{i d}+c_{2} P_{g d}\right) /\left(c_{1}+c_{2}\right)
$$

The procedure for implementing the QPSO is given by the following steps [11]:

Step 1. Initialization of population positions: Initialize a swarm of particles with random positions using a uniform probability distribution function.

Step 2. Evaluation of fitness function: Acquire the fitness value of each particle. 
Step 3. Comparison to pbest (personal best): Compare each particle's fitness with the particle's pbest in population.

Step 4. Comparison to gbest: Compare the fitness with the swarm's overall previous best.

Step 5. Updating of global point: Calculate the Mbest using equation (4).

Step 6. Updating of particles' position: Change the position of the particles according to equation (3), where $c_{1}$ and $c_{2}$ are two random numbers generated using a uniform probability distribution in the range $[0,1]$.

Step 7. Repeating the iteration cycle: Loop to Step 2 until a stop criterion is met, usually a sufficiently good fitness or a maximum number of iterations.

\section{Description of Case Study System}

Figure 1 is a single machine infinite bus power (SMIB) system installed with a STATCOM. The synchronous generator is delivering power to the infinite-bus through a double circuit transmission line and a STATCOM. The system data and STATCOM parameters are given in the Appendix A. The system consists of a step down transformer (SDT) with a leakage reactance $\mathrm{X}_{\mathrm{SDT}}$, a three phase GTO-based voltage source converter, and a de capacitor. The STATCOM model used in this study is found good enough for the low frequency oscillation stability problem [10].

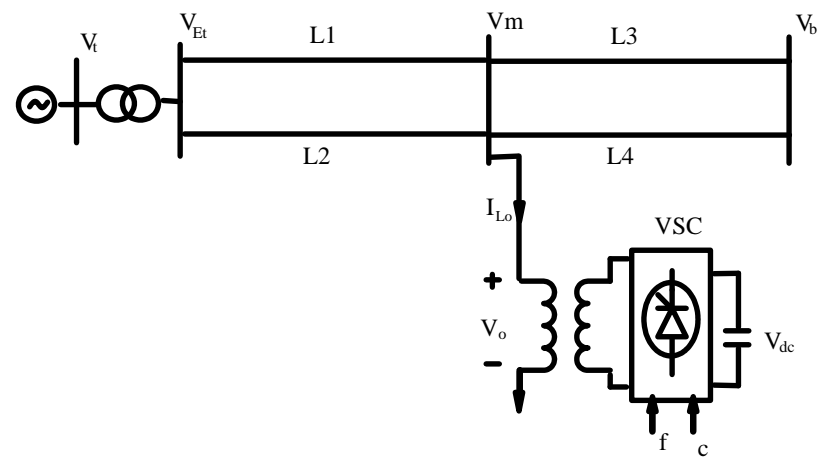

Figure 1. SMIB power system equipped with STATCOM

The VSC generates a controllable AC voltage source $v_{o}(t)=V_{0} \sin (w t-\varphi)$ behind the leakage reactance. The voltage difference between the STATCOM bus AC voltage, $\mathrm{v}_{\mathrm{L}}(\mathrm{t})$ and $\mathrm{v}_{0}(\mathrm{t})$ produces active and reactive power exchange between the STATCOM and the power system, which can be controlled by adjusting the magnitude $\mathrm{V}_{0}$ and the phase $\varphi$. The dynamic relation between the capacitor voltage and current in the STATCOM circuit are expressed as follows [5]:

$$
\begin{aligned}
& \bar{I}_{L o}=I_{L o d}+j I_{L o q} \\
& V_{o}=c V_{d c}(\cos \varphi+j \sin \varphi)=c V_{d c} \angle \varphi \\
& \dot{V}_{d c}=\frac{I_{d c}}{C_{d c}}=\frac{c}{C_{d c}}\left(I_{L o d} \cos \varphi+I_{L o q} \sin \varphi\right)
\end{aligned}
$$


Where, for the PWM inverter $c=m k$ and $\mathrm{k}$ is the ratio between $\mathrm{AC}$ and DC voltage depending on the inverter structure; $m$ is the modulation ratio defined by the PWM and the phase $c$ is also defined by the PWM. The $C_{d c}$ is the dc capacitor value and $I_{d c}$ is the capacitor current while $i_{\text {Lod }}$ and $i_{\text {Loq }}$ are the $d$-and $q$-components of the STATCOM current, respectively. The dynamics of the generator and the excitation system are expressed through a third order model given as [3]:

$$
\begin{aligned}
& \dot{\delta}=\omega_{0}(\omega-1) \\
& \dot{\omega}=\left(P_{m}-P_{e}-D \Delta \omega\right) / M \\
& \dot{E}_{q}^{\prime}=\left(-E_{q}+E_{f d}\right) / T_{d o}^{\prime} \\
& E_{f d}=\left(-E_{f d}+K_{a}\left(V_{r e f}-V_{t}\right)\right) / T_{a}
\end{aligned}
$$

The expressions for the power output, terminal voltage, and the d-q axes currents in the transmission line and STATCOM, respectively, are:

$$
\begin{aligned}
& P_{e}=V_{t d} I_{t d}+V_{t q} I_{t q} ; E_{q}=E_{q e}^{\prime}+\left(X_{d}-X_{d}^{\prime}\right) I_{t d} ; V_{t}=V_{t d}+j V_{t q} ; V_{t d}=X_{q} I_{t q} ; V_{t q}=E_{q}^{\prime}-X_{d}^{\prime} I_{t d} \\
& I_{t l d}=\frac{\left(1+\frac{X_{L B}}{X_{S D T}}\right) e_{q}^{\prime}-\frac{X_{L B}}{X_{S D T}} m V_{d c} \sin \varphi-V_{b} \cos \varphi}{X_{t L}+X_{L B}+\frac{X_{t L}}{X_{L B}}+\left(1+\frac{X_{L B}}{X_{S D T}}\right) x_{d}^{\prime}} \\
& I_{t l q}=\frac{\frac{X_{L B}}{X_{S D T}} m V_{d c} \cos \varphi+V_{b} \sin \varphi}{X_{t L}+X_{L B}+\frac{X_{t L}}{X_{L B}}+\left(1+\frac{X_{L B}}{X_{S D T}}\right) x_{q}} \\
& I_{L o d}=\frac{e_{q}^{\prime}-\left(x_{d}^{\prime}+X_{t L}\right) I_{t L q}-m V_{d c} \sin \varphi}{X_{S D T}} \\
& I_{L o q}=\frac{m V_{d c} \cos \varphi-\left(x_{d}^{\prime}+X_{t L}\right) I_{t L q}}{X_{S D T}}
\end{aligned}
$$

where, $X_{t L}=X_{T}+\frac{X_{L}}{2} ; X_{L B}=\frac{X_{L}}{2}, X_{T}, x_{d}^{\prime}$ and $x_{q}$ are the transmission line reactance, d-axis transient reactance, and q-axis reactance, respectively. A linear dynamic model is obtained by linearizing the nonlinear model round an operating condition. The linearized model of the power system shown in Figure 1 is given in Appendix B. The block diagram of the linearized dynamic model of the SMIB power system with STATCOM is shown in Figure 2.

\subsection{STATCOM based controllers}

The power oscillation damping (POD) controller is designed to produce an electrical torque in phase with the speed deviation according to phase compensation method. 


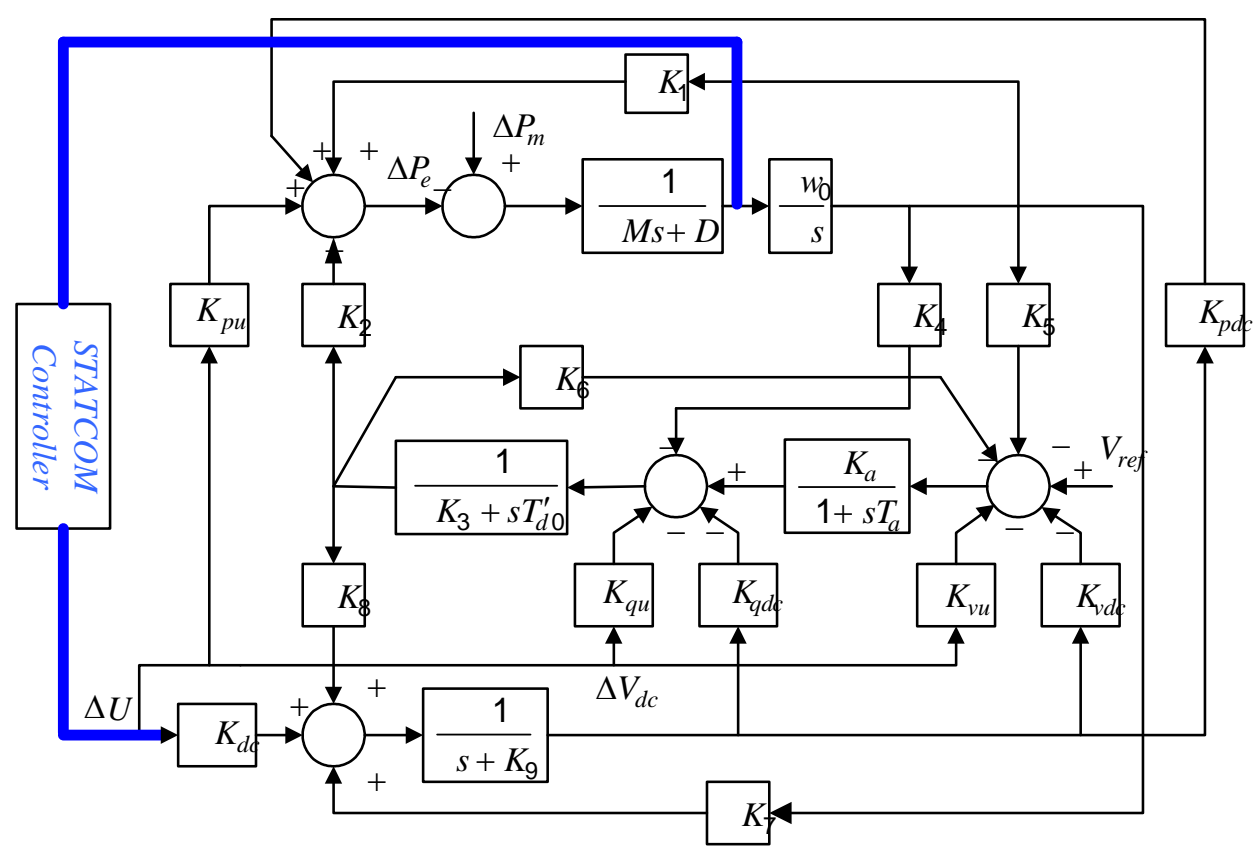

Figure 2. Modified Heffron-Phillips transfer function model

The speed deviation $\Delta \omega$ is considered as the input to the damping controller. The structure of POD controller is given in Figure 3. This controller may be considered as a lead-lag compensator [10]. However, an electrical torque in phase with the speed deviation is to be produced in order to improve damping of the system oscillations. It consists of a gain block, a signal-washout block and a lead-lag compensator. The block diagram of STATCOM dc voltage PI controller with power oscillation damping stabilizer is shown in Figure 4. The DCvoltage regulator controls the voltage across the DC capacitor of the STATCOM controller. Figure 5 illustrates the block diagram of STATCOM ac voltage PI controller with a power oscillation damping stabilizer.

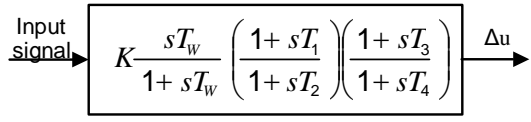

Figure 3. Power oscillation damping controller

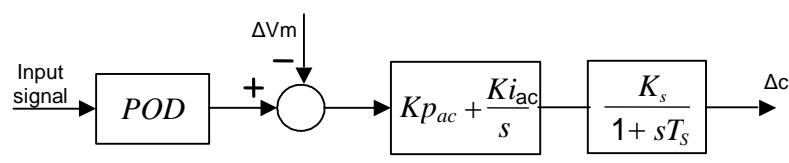

Figure 4. STATCOM PI controller for dc voltage

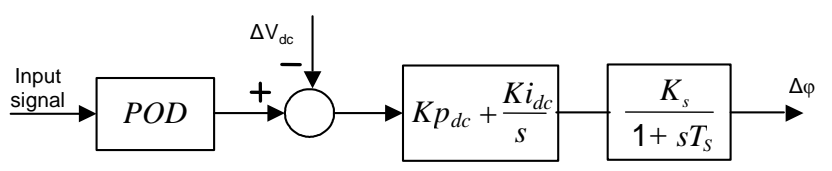

Figure 5. STATCOM PI controller for dc voltage 
$\mathrm{AC}$ voltage controller regulates the voltage of terminal according to requested reference that it accomplishes through changing of converter output voltage [3,10].

\subsection{STATCOM controller design using QPSO}

In the proposed method, we must tune the STATCOM controller parameters optimally to improve overall system dynamic stability in a robust way under different operating conditions and disturbances. A performance index based on the system dynamics after an impulse disturbance alternately occurs in the system is organized and used to form the objective function of the design problem. In this study, an Integral of Time multiplied Absolute value of the Error (ITAE) is taken as the objective function. Since the operating conditions in power systems are often varied, a performance index for a wide range of operating points is defined as follows [15]:

$J=\sum_{i=1}^{N p} \int_{0}^{t_{\text {sin }}}\left|\Delta \omega_{i}\right| \cdot t d t$

In the above equation, NP is the total number of operating points $\left(\mathrm{N}_{\mathrm{p}}=5\right)$ and $\mathrm{t}_{\mathrm{sim}}$ is the time range of simulation $\left(t_{\text {sim }}=8 \mathrm{sec}\right)$. For objective function calculation, the time-domain simulation of the power system model is carried out for the simulation period. The design problem can be formulated as the following constrained optimization problem, where the constraints are the controller parameters bounds:

Minimize J Subject to:

$$
\begin{aligned}
& K_{x}^{\min } \leq K_{x} \leq K_{x}^{\max } \\
& T_{x}^{\min } \leq T_{x} \leq T_{x}^{\max }
\end{aligned}
$$

The proposed approach employs QPSO to solve this optimization problem and search for an optimal or near optimal set of controller parameters. The optimization of controller parameters is carried out by evaluating the objective function as given in equation (17), which considers a multiple of operating conditions. The operating conditions are considered as:

- Base case: $\mathrm{P}=0.80 \mathrm{pu}, \mathrm{Q}=0.2 \mathrm{pu}$ and $\mathrm{X}_{\mathrm{L}}=0.4 \mathrm{pu}$.

(Nominal loading)

- Case 1: $\mathrm{P}=0.2 \mathrm{pu}, \mathrm{Q}=0.01$ and $\mathrm{X}_{\mathrm{L}}=0.4 \mathrm{pu}$

(Light loading)

- Case 2: $\mathrm{P}=1.20 \mathrm{pu}, \mathrm{Q}=0.4$ and $\mathrm{X}_{\mathrm{L}}=0.4 \mathrm{pu}$.

(Heavy loading)

- Case 3: The $20 \%$ increase of line reactance $X_{L}$ at nominal loading condition.

- Case 4: The $20 \%$ increase of line reactance $X_{L}$ at heavy loading condition.

In this work, in order to acquire better performance, number of particles, particle size, number of iterations and $\beta$ is chosen as $30,7,50$ and 1.5 , respectively. It should be noted that QPSO algorithm is run several times and then optimal set of STATCOM controller parameters is selected. The performance of the proposed method is compared to that of classical PSO based STATCOM controller, the GA method given in [10] and the phase compensation method [3]. The final values of the optimized parameters with objective function, $J$, are given in Table 1. Also, Figure 6 shows the minimum fitness functions evaluating process. 

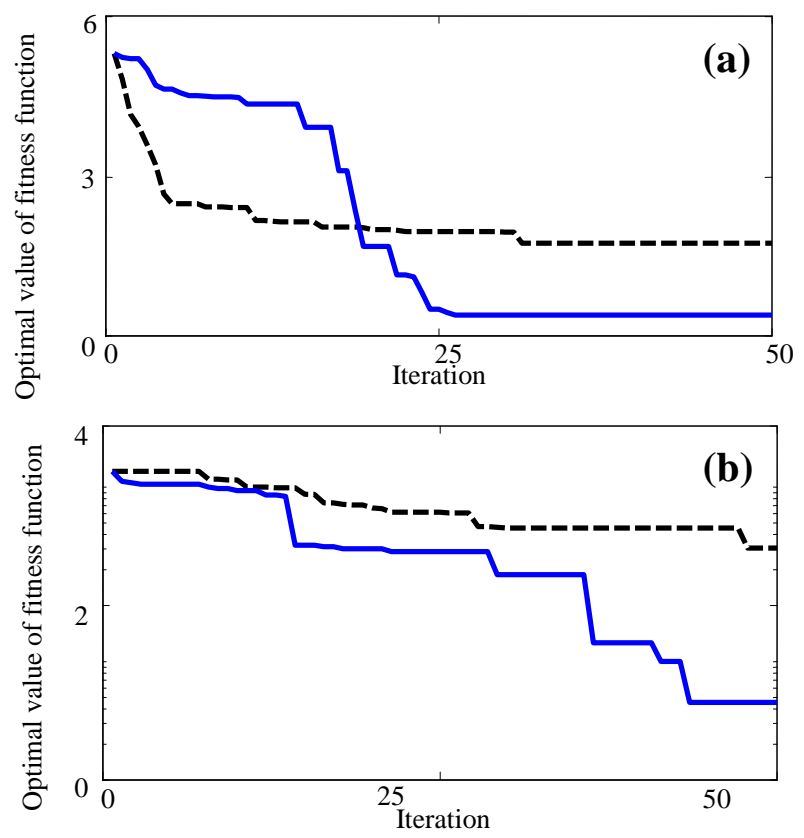

Figure 6. The convergence ratio of the fitness function: (a) $\varphi$ based controller (b) C based controller; Solid (QPSO), Dashed (CPSO)

Table 1. The optimal parameter settings of the proposed controllers

\begin{tabular}{|c|c|c|c|c|c|c|c|c|c|c|}
\hline $\begin{array}{c}\text { Type of } \\
\text { algorithm }\end{array}$ & $\begin{array}{l}\text { Type of } \\
\text { controller }\end{array}$ & K & $\mathrm{T}_{1}$ & $\mathrm{~T}_{2}$ & $\mathrm{~T}_{3}$ & $\mathrm{~T}_{4}$ & $\mathrm{Kp}_{\mathrm{dc}}$ & $\mathrm{Ki}_{\mathrm{dc}}$ & $\mathrm{Kp}_{\mathrm{ac}}$ & $\mathrm{Ki}_{\mathrm{ac}}$ \\
\hline \multirow{2}{*}{$\begin{array}{c}\text { Phase } \\
\text { Compensation }\end{array}$} & $\mathrm{C}$ & 99.43 & 0.6454 & 0.7734 & 0.1891 & 0.3909 & --- & --- & 1.321 & 0.049 \\
\hline & $\varphi$ & 120.00 & 0.0444 & 0.7346 & 0.8339 & 0.7441 & 140.00 & 0.100 & --- & --- \\
\hline & $\mathrm{C}$ & 118.84 & 0.1881 & 0.8973 & 0.7658 & 0.4302 & --- & --- & 1.048 & 0.057 \\
\hline & $\varphi$ & 151.23 & 0.2363 & 0.7790 & 0.3143 & 0.836 & 123.65 & 0.085 & --- & --- \\
\hline \multirow{2}{*}{ CPSO } & $\mathrm{C}$ & 98.54 & 0.375 & 0.8765 & 0.8889 & 0.4361 & --- & --- & 2.276 & 0.026 \\
\hline & $\varphi$ & 181.44 & 0.3633 & 0.6796 & 0.1143 & 0.9368 & 101.48 & 0.4852 & --- & --- \\
\hline \multirow{2}{*}{ QPSO } & $\mathrm{C}$ & 197.06 & 0.3957 & 1.4967 & 0.3330 & 0.9902 & --- & --- & 9.76 & 0.958 \\
\hline & $\varphi$ & 193.23 & 0.065 & 0.674 & 0.869 & 0.6978 & 151.32 & 0.213 & --- & --- \\
\hline
\end{tabular}

\section{Nonlinear Time Domain Simulation}

To investigate the performance of designed controllers, two classes of disturbances are studied. These classes are chosen to represent the large, as well as small power system disturbances.

\subsection{Response to small disturbance}

To assess the performance of the proposed method, a disturbance of $0.2 \mathrm{pu}$ input torque is applied to the machine at $t=1 \mathrm{sec}$. The study is performed at three different operating conditions. The results are shown in Figures 7 and 8. It can be seen that the QPSO based STATCOM power oscillation damping controller achieves good robust performance and enhances greatly the dynamic stability of power systems. It can be seen that the QPSO-based stabilizer provides better damping characteristics and enhances greatly the first swing stability compared to that of PSO, GA and phase compensation based stabilizers. 


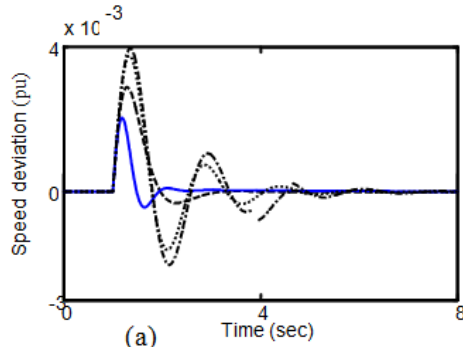

(a)

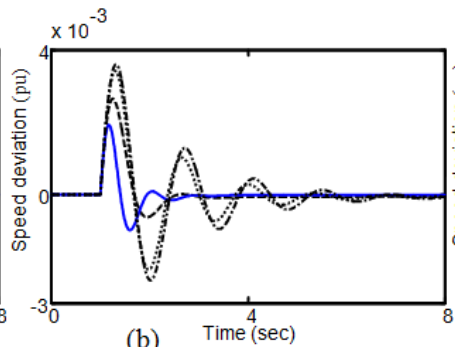

(b) Time (sec)

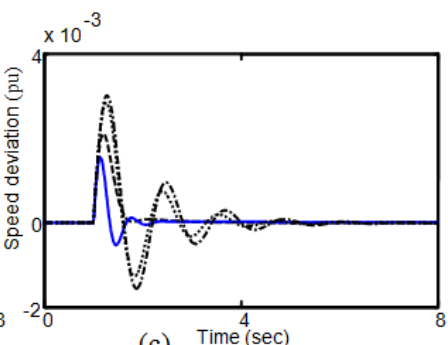

(c) Time (sec)

Figure 7. Dynamic responses for $\Delta \omega$ with $C$ based controller at (a) nominal (b) light and (c) heavy loading; Solid (QPSO), Dashed (CPSO), Dotted (GA [10]) and Dash-Dotted

(Phase compensation method [3])
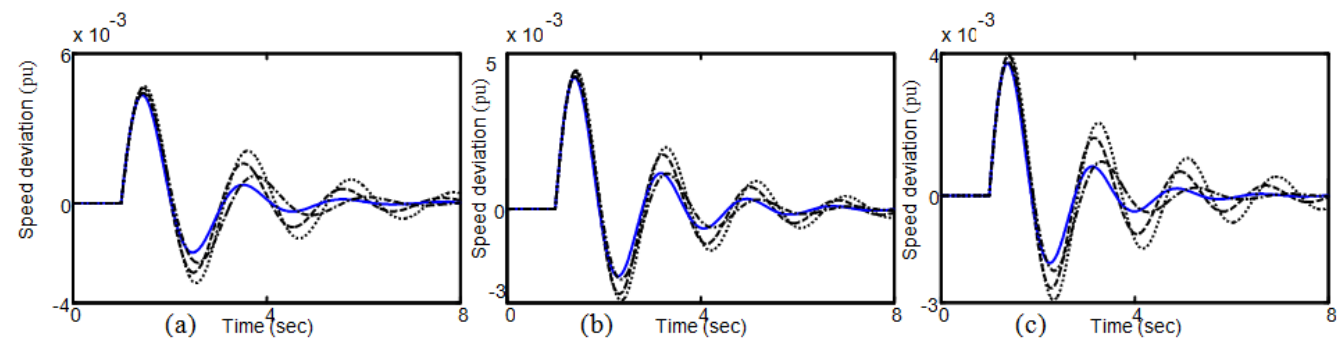

Figure 8. Dynamic responses for $\Delta \omega$ with $\varphi$ based controller at (a) nominal (b) light and (c) heavy loading; Solid (QPSO), Dashed (CPSO), Dotted (GA [10]) and Dash-Dotted (Phase compensation method [3]).
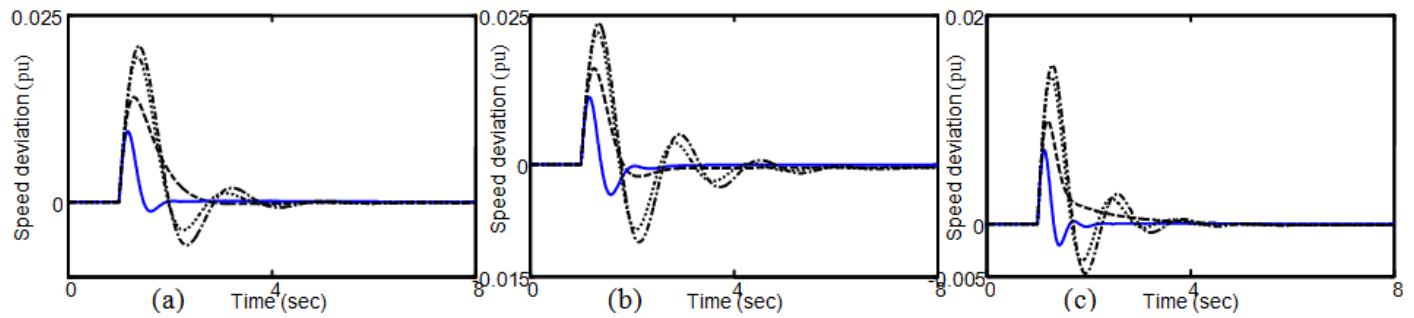

Figure 9. Dynamic responses for $\Delta \omega$ with $\mathrm{C}$ based controller at (a) nominal (b) light and (c) heavy loading; Solid (QPSO), Dashed (CPSO), Dotted (GA [10]) and Dash-Dotted (Phase compensation method [3]).
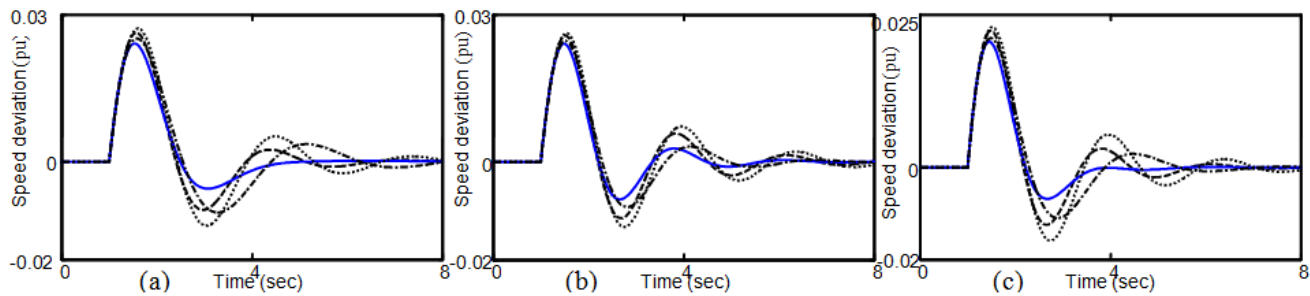

Figure 10. Dynamic responses for $\Delta \omega$ with $\varphi$ based controller at (a) nominal (b) light and (c) heavy loading; Solid (QPSO), Dashed (CPSO), Dotted (GA [10]) and Dash-Dotted (Phase compensation method [3]). 


\subsection{Response to a large disturbance}

In this section, the performance of the proposed controller under transient conditions is verified by applying a 6-cycle three-phase fault at $t=1 \mathrm{sec}$, at the middle of the transmission line $L_{3}$. The fault is cleared by permanent tripping of the faulted line. The system response to this disturbance is shown in Figures 9 and 10. It can be seen that the proposed controller has a good performance in damping low frequency oscillations and stabilizes the system quickly.

To demonstrate the performance of robustness of the proposed method, two performance indices: The Integral of the Time multiplied Absolute value of the Error (ITAE) and Figure of Demerit (FD) based on the system performance characteristics are defined as [15]:

$$
\begin{aligned}
& I T A E=1000 \int_{0}^{t s i m}|\Delta \omega| \cdot t d t \\
& F D=(O S \times 1000)^{2}+(U S \times 4000)^{2}+T_{s}^{2}
\end{aligned}
$$

Where, speed deviation, $(\Delta \omega)$, Overshoot (OS), Undershoot (US) and settling time of speed deviation of the machine are considered for evaluation of the ITAE and FD indices. It is worth mentioning that the lower the value of these indices is, the better the system response in terms of time-domain characteristics. Numerical results for all system loading cases are presented in Tables 2 and 3. This demonstrates that the overshoot, undershoot, settling time and speed deviations of the machine are greatly reduced by applying the proposed QPSO based tuned controllers.

Table 2. Values of Performance Index ITAE

\begin{tabular}{|c|c|c|c|c|c|c|c|c|c|c|}
\hline \multirow{2}{*}{$\begin{array}{c}\text { Type of } \\
\text { disturbance }\end{array}$} & Type of & \multicolumn{3}{|c|}{ Nominal } & \multicolumn{3}{c|}{ Light } & \multicolumn{3}{c|}{ Heavy } \\
\cline { 3 - 11 } & controller & GA & CPSO & QPSO & GA & CPSO & QPSO & GA & CPSO & QPSO \\
\hline \multirow{2}{*}{ Small } & C & 6.2912 & 2.726 & 1.285 & 7.339 & 3.00 & 1.502 & 3.696 & 1.209 & 0.7628 \\
\cline { 2 - 12 } & $\varphi$ & 27.976 & 20.04 & 11.043 & 22.48 & 18.565 & 11.779 & 24.081 & 17.049 & 8.783 \\
\hline \multirow{2}{*}{ Large } & $\mathrm{C}$ & 4.771 & 3.494 & 0.9709 & 6.576 & 3.331 & 1.323 & 2.73 & 1.973 & 0.5555 \\
\cline { 2 - 11 } & $\varphi$ & 23.656 & 16.123 & 11.791 & 22.554 & 17.753 & 11.092 & 18.215 & 12.346 & 7.466 \\
\hline
\end{tabular}

Table 3. Values of Performance Index FD

\begin{tabular}{|c|c|c|c|c|c|c|c|c|c|c|}
\hline \multirow{2}{*}{$\begin{array}{c}\text { Type of } \\
\text { disturbance }\end{array}$} & Type of & \multicolumn{3}{|c|}{ Nominal } & \multicolumn{3}{c|}{ Light } & \multicolumn{3}{c|}{ Heavy } \\
\cline { 3 - 11 } & controller & GA & CPSO & QPSO & GA & CPSO & QPSO & GA & CPSO & QPSO \\
\hline \multirow{2}{*}{ Small } & $\mathrm{C}$ & 76.52 & 18.04 & 10.73 & 105.49 & 31.14 & 26.18 & 47.76 & 11.19 & 10.25 \\
\cline { 2 - 12 } & $\varphi$ & 240.69 & 195.63 & 116.39 & 212.42 & 189.66 & 129.88 & 207.43 & 186.05 & 97.91 \\
\hline \multirow{2}{*}{ Large } & $\mathrm{C}$ & 35.72 & 14.47 & 7.81 & 91.56 & 27.14 & 19.87 & 23.80 & 13.12 & 7.17 \\
\cline { 2 - 11 } & $\varphi$ & 201.23 & 128.56 & 63.42 & 188.91 & 169.28 & 89.68 & 174.52 & 115.36 & 46.337 \\
\hline
\end{tabular}

\section{Conclusions}

A method of designing a power oscillation damping controller for a STATCOM has been proposed. The design problem of the controller is converted into an optimization problem which is solved by a QPSO technique with the time domain-based objective function. The controller was tested for a number of disturbance conditions including small and large disturbances. The robust design has been found to be very effective for a wide range of operating conditions. The non-linear time domain simulation results and system performance characteristics show that designed QPSO based STATCOM damping controller has an excellent capability in damping low frequency oscillations in comparison with designed classical PSO and genetic algorithm (GA) based STATCOM controllers. 


\section{APPENDIX A}

The nominal parameters of the system are listed in Table 4.

Table 4. System parameters

\begin{tabular}{|c|c|c|c|}
\hline \multirow{2}{*}{ Generator } & $M=8 \mathrm{MJ} / \mathrm{MVA}$ & $T_{d o}^{\prime}=5.044 \mathrm{~s}$ & $X_{d}=1 \mathrm{pu}$ \\
\hline & $X_{q}=0.6 \mathrm{p} . \mathrm{u}$ & $X_{d}^{\prime}=0.3 \mathrm{pu}$ & $D=0$ \\
\hline \multicolumn{2}{|c|}{ Excitation system } & $K_{a}=50$ & $T_{a}=0.05 \mathrm{~s}$ \\
\hline \multicolumn{2}{|c|}{ Transformers } & $X_{T}=0.1 p u$ & $X_{S D T}=0.1 p u$ \\
\hline \multicolumn{2}{|c|}{ Transmission line } & $X_{q}=0.4 \mathrm{pu}$ & \\
\hline \multicolumn{2}{|c|}{ DC link parameter } & $V_{D C}=1 p u$ & $C_{D C}=1 p u$ \\
\hline \multirow{2}{*}{\multicolumn{2}{|c|}{ STATCOM parameter }} & $C=0.25$ & $\varphi=52^{\circ}$ \\
\hline & & $\mathrm{K}_{\mathrm{s}}=1$ & $\mathrm{~T}_{\mathrm{S}}=0.05$ \\
\hline
\end{tabular}

Appendix B: The linearized system equations

Taking variations around the operating points, equations (9)-(12) can be written as:

$$
\begin{aligned}
& \Delta \dot{\delta}=\omega_{0} \Delta \omega \\
& \Delta \dot{\omega}=\left(-\Delta P_{e}-D \Delta \omega\right) / M \\
& \dot{E}_{q}^{\prime}=\left(-\Delta E_{q}+\Delta E_{f d}\right) / T_{d o}^{\prime} \\
& \Delta \dot{E}_{f d}=\left(K_{A}\left(\Delta v_{r e f}-\Delta v\right)-\Delta E_{f d}\right) / T_{A} \\
& \Delta \dot{v}_{d c}=K_{7} \Delta \delta+K_{8} \Delta E_{q}^{\prime}-K_{9} \Delta v_{d c}+K_{d c} \Delta c+K_{d \varphi} \Delta \varphi \\
& \Delta P_{e}=K_{1} \Delta \delta+K_{2} \Delta E_{q}^{\prime}+K_{p d c} \Delta v_{d c}+K_{p c} \Delta c+K_{p \varphi} \Delta \varphi \\
& \Delta E_{q}^{\prime}=K_{4} \Delta \delta+K_{3} \Delta E_{q}^{\prime}+K_{q d c} \Delta v_{d c}+K_{q c} \Delta c+K_{q \varphi} \Delta \varphi \\
& \Delta V_{t}=K_{5} \Delta \delta+K_{6} \Delta E_{q}^{\prime}+K_{v d c} \Delta v_{d c}+K_{v c} \Delta c+K_{v \varphi} \Delta \varphi
\end{aligned}
$$

$K_{1}, K_{2} \ldots K_{9}, K_{p u}, K_{q u}$ and $K_{v u}$ are linearization constants. The state-space model of the power system is given by:

$\dot{x}=A x+B u$

Where, the state vector $x$, control vector $u, A$ and $B$ are:

$$
\begin{aligned}
& x=\left[\begin{array}{llllll}
\Delta \delta & \Delta \omega & \Delta E_{q}^{\prime} & \Delta E_{f d} & \Delta v_{d c}
\end{array}\right]^{T} ; u=\left[\begin{array}{ll}
\Delta c & \Delta \varphi
\end{array}\right]^{T} \\
& A=\left[\begin{array}{ccccc}
0 & w_{0} & 0 & 0 & 0 \\
-\frac{K_{1}}{M} & 0 & -\frac{K_{2}}{M} & 0 & -\frac{K_{p d c}}{M} \\
-\frac{K_{4}}{T_{d o}^{\prime}} & 0 & -\frac{K_{3}}{T_{d o}^{\prime}} & -\frac{1}{T_{d o}^{\prime}} & -\frac{K_{q d c}}{T_{d o}^{\prime}} \\
-\frac{K_{A} K_{5}}{T_{A}} & 0 & -\frac{K_{A} K_{6}}{T_{A}} & -\frac{1}{T_{A}} & -\frac{K_{A} K_{v d c}}{T_{A}} \\
K_{7} & 0 & K_{8} & 0 & -K_{9}
\end{array}\right] ; \quad B=\left[\begin{array}{cc}
0 & 0 \\
-\frac{K_{p c}}{M} & -\frac{K_{p \varphi}}{M} \\
-\frac{K_{q c}}{T_{d o}^{\prime}} & -\frac{K_{q \varphi}}{T_{d o}^{\prime}} \\
-\frac{K_{A} K_{v c}}{T_{A}} & -\frac{K_{A} K_{v \varphi}}{T_{A}} \\
K_{d c} & K_{d \varphi}
\end{array}\right]
\end{aligned}
$$




\section{References}

[1] J. Machowski and J. W Bialek, "state variable control of shunt FACTS devices using phasor measurements", Elect. Power Syst Res., vol. 78, (2008), pp. 39-48.

[2] N. G. Hingorani and L. Gyugyi, "Understanding FACTS: concepts and technology of flexible AC transmission systems", Wiley-IEEE Press, (1999).

[3] H. F. Wang, "Phillips-Heffron model of power systems installed with STATCOM and applications", IEE Proc. Gen. Transm. Dist., vol. 146, no. 5, (1999), pp. 521-527.

[4] S. Morris, P. K. Dash and K. P. Basu, "A fuzzy variable structure controller for STATCOM", Elect Power Syst Res., vol. 65, (2003), pp. 23-34.

[5] A. H. M. A. Rahim and M. F. Kandlawala, "Robust STATCOM voltage controller design using loop shaping technique", Elect. Power Syst Res., vol. 68, (2004), pp. 61-74.

[6] A. H. M. A. Rahim, S. A. Al-Baiyat and H. M. Al-Maghrabi, "Robust damping controller design for a static compensator", IEE. Proc-Gener. Transm. Distrib., vol. 149, no. 4, (2002), pp. 491-496.

[7] M. Clerc and J. Kennedy, "The particle swarm-explosion, stability, and convergence in a multidimensional complex space”, IEEE Trans. Evol Comput, vol. 6, no. 1, (2002), pp. 58-73.

[8] R. Poli, J. Kennedy and T. Blackwell, "Particle swarm optimization: An overview", Swarm Intelligent., vol. 1, (2007), pp. 33-57.

[9] H. Shayeghi, H. A. Shayanfar, S. Jalilzadeh and A. Safari, "A quantum particle swarm optimizer for the tuning of Unified Power Flow Controller”, Energy Conver. Manage., vol. 51, (2010), pp. 2299-2306.

[10] M. A. Abido, "Analysis and assessment of STATCOM based damping stabilizers for power system stability enhancement”, Elect. Power Syst Res., vol. 73, (2005), pp. 177-185.

[11]L. S Coelho, "A quantum particle swarm optimizer with chaotic mutation operator", Chaos Soliton. Fractals., vol. 37, (2008), pp. 1409-1418.

[12] J. Liu, W. Xu and J. Sun, "Quantum-behaved particle swarm optimization with mutation operator", International conference on tools with artificial intelligence, Hong Kong (China), (2005).

[13] J. Sun, W. Fang, W. Chen and W. Xu, "Design of two dimensional IIR digital filters using an improved quantum-behaved particle swarm optimization algorithm”, in Proceedings of American Control conference, (2008), pp. 2603-2608.

[14] J. Sun, W. Fang and W. Xu, "Adaptive parameter control for quantum-behaved particle swarm optimization on individual level", IEEE international conference on systems, man and cybernetics, Big Island, USA; (2005), pp. 3049-3054.

[15] A. Safari, “ $\Theta-P S O$ Algorithm for UPFC Based Output Feedback Damping Controller”, International Journal of Control and Automation, vol. 6, no. 1, (2013), pp. 33-46.

\section{Author}

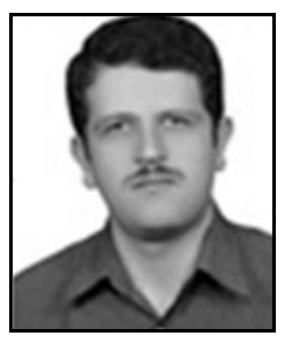

\section{Amin Safari}

He received the B.Sc. and M.Sc. degrees in Electrical Engineering in 2007 and 2009, respectively. He received his Ph.D. degree in Power Electrical Engineering, Iran University of Science and Technology, Tehran, Iran. Currently, he is an Assistant Professor in Department of Electrical Engineering, Azarbaijan Shahid Madani University, Tabriz, Iran. His areas of interest in research are Application of artificial intelligence to power system control design, FACTS device and fuzzy sets and systems. He has published more than 70 papers in international journals and conference proceedings. 